\title{
Discours
}

Revue de linguistique, psycholinguistique et informatique. A journal of linguistics, psycholinguistics and computational linguistics

14 | 2014

Varia

\section{La focalisation des conditionnelles}

\section{Gilles Corminboeuf}

\section{OpenEdition}

Journals

\section{Édition électronique}

URL : http://journals.openedition.org/discours/8903

DOI : 10.4000/discours.8903

ISSN : 1963-1723

Éditeur :

Laboratoire LATTICE, Presses universitaires de Caen

\section{Référence électronique}

Gilles Corminboeuf, "La focalisation des conditionnelles », Discours [En ligne], 14 | 2014, mis en ligne le 16 septembre 2014, consulté le 02 mai 2019. URL : http://journals.openedition.org/discours/8903 ; DOI : 10.4000/discours.8903

\section{(c) (i) (2)}

Discours est mis à disposition selon les termes de la licence Creative Commons Attribution - Pas d'Utilisation Commerciale - Pas de Modification 4.0 International. 

Revue de linguistique, psycholinguistique et informatique

\section{La focalisation des conditionnelles}

Gilles Corminboeuf

Fonds national suisse de la recherche scientifique

Gilles Corminboeuf, «La focalisation des conditionnelles», Discours [En ligne], 14 | 2014, mis en ligne le 16 septembre 2014 .

URL: http://discours.revues.org/8903

Titre du numéro: Varia

Coordination: Laure Sarda et Denis Vigier 



\title{
La focalisation des conditionnelles
}

\author{
Gilles Corminboeuf
}

Fonds national suisse de la recherche scientifique

L'objectif de cet article est d'identifier les différents moyens syntaxiques dont dispose le français pour focaliser les circonstants en si, dans des constructions du type $\{s i P, Q\}$ et du type $\{Q$ si $P\}$. Considérer que le constituant si $P$ puisse occuper une position de focus n'est pas si anodin, puisqu'on tient généralement pour acquis depuis Haiman (1978) que «conditionals are topics». La littérature scientifique s'est peu intéressée aux cas où si $P$ a un statut informationnel de focus. L'analyse porte sur trois classes de faits où la proposition si P est promue informationnellement: d'une part, des propositions si P placées dans le champ d'un opérateur (négation, restriction) ou dans un dispositif (clivage, pseudo-clivage). D'autre part, des propositions si $P$ en contexte d'enchâssement. Enfin, des propositions si P orphelines de leur terme $Q$, comme «Et si on allait au cinéma?». D'un point de vue syntaxique, si $P$ implique la présence de $Q$, mais d'un point de vue informationnel, la situation s'inverse: une contrainte d'informativité rend l'élément si $P$ inéluctable. L'étude montre qu'il existe des circonstants en si qui ont systématiquement un statut de topique (les conditionnelles dites «factuelles») et qu'il existe également des circonstants en si qui ont toujours un statut de focus (certaines propositions si $P$ comparatives, enchâssées, ou présentant une ellipse de Q).

Mots clés: conditionnelles, focus, topique, enchâssement, ellipse

The aim of this article is to identify the different syntactic modes existing in French to focus if-clauses in constructions such as \{if-P, Q $\}$, and $\{\mathrm{Q}$ if- $\mathrm{P}\}$. It is not irrelevant to consider that if-clauses may occupy a focus position, seeing that since Haiman (1978), it is generally admitted that "conditionals are topics". Little attention has been paid in the literature, however, to contexts where if-P has the information status of focus. The present analysis concerns three categories of facts where if-P has a status of focus: if-clauses located within the scope of a negation or a restriction, or in cleft or pseudocleft constructions; if-clauses in an embedding context; and lastly, ellipsis of the Q component, as in "Et si on allait au cinéma?" [What if we went to the movies?]. From a syntactic point of view, an if-clause demands a $Q$ component, but from an informational perspective, the reverse holds: an informative constraint makes if-P mandatory. The study demonstrates the existence of if-clauses which have systematically a topic status (so-called "factual" if-clauses) and of if-clauses which have a focus status (some comparative if-clauses, either embedded or with ellipsis of $Q$ ).

Keywords: conditionals, focus, topic, embedding, ellipsis

\section{Introduction}

On considère généralement comme acquis depuis Haiman (1978) que «conditionals are topics», mais le français, comme d'autres langues, dispose de procédés variés pour focaliser les segments si $P$. Dans une perspective typologique, Haiman observe des convergences formelles entre les conditionnelles et les topiques. Les trois arguments centraux de l'auteur sont les suivants: (i) dans certaines langues, une particule homonyme marque à la fois les topiques et les conditionnelles: "Conditional clauses and topics are marked identically in a number of unrelated languages» (Haiman, 1978 : 564); (ii) d'un point de vue iconique, topiques et conditionnelles sont généralement 
des constituants frontaux (Haiman, 1978: 572); (iii) les définitions ordinaires des topiques et des conditionnelles permettent de les associer à ce qui est de l'ordre du «donné». Cette assimilation entre protase conditionnelle et fonction de topique est discutée par de nombreux spécialistes, par exemple Ford et Thompson (1986), Comrie (1986), Akatsuka (1986), Sweetser (1990) et Charolles (2003).

Cet article est consacré à l'étude des différents moyens syntaxiques dont dispose le français pour focaliser les circonstants en $s i$. J'analyse trois classes de faits apparemment très différentes, mais qui ont partie liée avec la promotion informationnelle de si $P$ dans des constructions en $\{$ si $P, Q\}$ et en $\{Q$ si $P\}$ :

- des propositions si $P$ placées dans le champ d'un opérateur ou dans un dispositif (partie I);

- des propositions si $P$ en contexte d'enchâssement (partie 2);

- des propositions si $P$ «orphelines» de leur terme $\mathrm{Q}$ (partie 3 ).

Les si $P$ enchâssées (partie 2) seront étudiées essentiellement parce qu'elles révèlent des contraintes spécifiques. Leur capacité à être enchâssées est plus une condition pour être focalisées qu'un moyen de l'être. Les contextes d'enchâssement me permettront également de clarifier la question de la position de si $P$, de son statut syntaxique et de son détachement par rapport à $\mathrm{Q}^{1}$. Bien que je n'introduise pas les principes d'un modèle informationnel en particulier, ma conception de l'opposition entre topique et focus est largement inspirée (mais librement adaptée) de la distinction de Kronning (1996: 45 sq.) entre substrat et foyer. Je définis le focus comme l'information présentée comme nouvelle et pertinente à un moment donné du discours; morphosyntaxiquement, le focus est le segment qui est placé sous la portée des opérateurs propositionnels ou qui entre dans des dispositifs relevant de la rection verbale, comme le clivage. J'assigne le statut de topique aux segments positionnés hors focus.

Les exemples utilisés dans cet article sont constitués de données orales (transcriptions radiophoniques ou télévisuelles), d'éléments écrits issus de la presse écrite, d'œuvres littéraires diverses (classiques et contemporaines), de discours scientifiques, de données issues de la base textuelle Frantext ${ }^{2}$, du web et du télétexte ${ }^{3}$.

1. Je laisserai de côté le rôle de la prosodie dans le marquage de la structure informationnelle. Le placement de si $P$ sous intonème conclusif peut être un moyen de réaliser une focalisation: «Le morphème intonatif conclusif CC est l'opérateur de rhématisation. [...] Le rhème est constitué des éléments syntaxiques qui demeurent devant le morphème intonatif conclusif majeur CC» (Rossi, I999: II5). Cornulier (I985: 79) souligne qu'un accent d'insistance sur le relateur si peut opérer un marquage focal: «On ira au cinéma si on ne va pas à la plage».

2. ATILF. Base textuelle Frantext. En ligne à l'adresse suivante: http://www.frantext.fr/.

3. Cette étude s'appuie particulièrement sur un corpus qui a été exploité dans plusieurs travaux de plus grande ampleur, notamment Corminboeuf (2009). Les données le constituant ont été collectées entre 2004 et 2007 et sont à la base de ma thèse de doctorat intitulée L'expression de l'bypothèse en français contemporain, entre bypotaxe et parataxe (université de Neuchâtel, 2008). Voir : http://doc.rero.ch/record/943o. Le corpus est en accès libre à l'adresse suivante : http://doc.rero.ch/record/943o/files/th_CorminboeufGAnnexes.pdf. Les exemples extraits de ce corpus porteront, dans cet article, la mention «Corminboeuf - Corpus thèse». 


\section{Les opérateurs focalisants}

Dans cette partie initiale, j'exposerai deux types de moyens pour focaliser si $P$ : placer le constituant à l'intérieur d'un «dispositif» (I.I) ou dans le champ d'un opérateur (I.2 et I.3).

\subsection{Insérer si $P$ dans un dispositif}

Le clivage en [I] et le pseudo-clivage en [2] permettent de focaliser l'élément si P:

[Ia] On ira si je veux, gueula la mère, c'est si je le veux seulement qu'on ira!

(Frantext: Marguerite Duras, Un barrage contre le Pacifique [1950], Paris, Gallimard, 1994, p. IOI)

[Ib] C'est surtout $s i$ les États-Unis ne sont pas représentés à Genève qu'ils prennent des risques.

(Frantext: Pierre Mendès-France, Euvres complètes, Paris, Gallimard, t. III, Gouverner, c'est choisir, 1954-1955, 1986, p. I2I)

[2] là où ç'aurait été intéressant c'est sỉls nous avaient dit au début qu'il fallait lire le journal en continu

(Corminboeuf - Corpus thèse: oral)

Les propositions clivées de Duras en [ia] font figure de rareté. En français, le clivage de si $P$ s'accompagne presque systématiquement d'un adverbe qui précède $s i$, comme surtout en [Ib], seulement ou uniquement; dans l'exemple [ia], l'adverbe seulement est placé après le syntagme verbal. La construction la mieux attestée serait donc: «c'est seulement si je le veux qu'on ira». Le clivage de si $P$ sans l'intercalation d'un de ces adverbes est très peu représenté en français ${ }^{4}$. Dans l'exemple [2], l'élément si $P$ entre dans un dispositif pseudo-clivé. Celui-ci n'est réputé compatible qu'avec les interrogatives indirectes totales (Delaveau, 1980). Pourtant, dans [2], il est plus plausible de considérer que si $P$ est un circonstant. Il est en effet difficile d'insérer une disjonction comme ou pas (propre aux interrogatives indirectes totales) alors qu'une commutation de $s i$ avec un autre introducteur de circonstant comme au cas où est réalisable. On notera que le dispositif pseudo-clivé ne réclame pas la présence d'un adverbe restrictif ou scalaire, comme c'est le cas pour le clivage.

\subsection{Insérer si $P$ dans le champ d'un opérateur}

8 La restriction et la négation sont des opérateurs qui ont le focus sous leur portée.

\subsubsection{La restriction}

9 Dans les exemples [3] et [4], l'élément si $P$ est placé dans le champ d'un opérateur restrictif:

4. Une recherche sur Frantext et sur le web a confirmé la chose. 
[3] La contrainte pragmatique s'appliquant à l'usage mathématique impose alors qu'on ne peut, dans un discours mathématique, présenter $\mathrm{X}$ comme argument pour $\mathrm{Y}$ que si $\mathrm{X}$ implique $\mathrm{Y}$.

(Corminboeuf - Corpus thèse : discours scientifique)

[4] Si l'on adopte le point de vue que la description historique d'une personne et de son œuvre n'a d'intérêt que s'il a contribué à l'avancement de la science, alors toute cette étude sur Forsskal n'a pas grande valeur...

(Corminboeuf - Corpus thèse: Thorkild Hansen, La mort en Arabie)

Dans l'exemple [3], la proposition si $P$ est dans le champ de l'opérateur restrictif. Dans [4], la subordonnée est stratifiée : l'élément si $P$ antéposé contient lui-même un constituant si $P$ positionné dans le champ de la restriction $n(e) \ldots q$ que. Le second élément si $P$ («s'il a contribué [...]») occupe une zone focale à l'intérieur de la cadrative détachée («Si l'on adopte [...]») qui a une fonction de topique.

\subsubsection{La négation}

Dans l'exemple [5], les parenthèses angulaires délimitent l'opérande de la négation. Le focus est situé à l'intérieur de cet opérande:

[5] Bon, je dirai un mot à Bernard en passant, sans insister. Sans grand effet, je pense. Mais il est trop malin pour ne pas < surveiller sa conduite, si elle compromet son prestige dans la maison $>$.

(Jacques Mercanton, L'été des Sept-Dormants, Lausanne, L'Âge d'Homme, I980, p. 156$)$

Les éléments si $P$ des exemples [3] à [5] sont positionnés à droite du verbe recteur, mais sont intégrés (et non détachés); à l'oral, le liage peut se réaliser au moyen de la prosodie. Dans l'exemple [5], la négation réalise une intégration syntaxique de si $P$ à l'intérieur du prédicat de Q: la syntaxe rend en quelque sorte la présence de la virgule non pertinente.

L'exemple [6] présente un contraste de focus articulé par le connecteur mais, le second élément si $P$ étant placé dans le champ de la négation:

[6] - Mais enfin, tu sais bien que je ne peux pas aller raconter des histoires pareilles à des cognes, quoi! On serait tous dans le coup.

- Si tu racontes tout, oui, mais pas si tu te contentes de les avertir.

- Mais tu les connais pas, je te dis; si tu avais vu la tête de Ferdinand Magnin quand il est sorti de leurs pattes [...].

(Frantext: Bernard Clavel, Malataverne [1960], Paris, J’ai Lu, 1990, p. I20)

Dans la seconde réplique de [6], le constituant si $P(«$ Si tu racontes tout» / «si tu te contentes de les avertir») est deux fois successivement présenté comme contenant le focus. 
Söhrman (2002: 20I) observe dans un corpus d'espagnol écrit que la négation affecte $\mathrm{P}$ dans $13 \%$ des cas lorsque si $P$ est antéposé et dans $26 \%$ lorsque si $P$ est placé à droite, ce qui tendrait à démontrer une affinité entre la position droite et le caractère focalisé. Resterait cependant à savoir si ces éléments si $P$ sont intégrés ou détachés, ce qui constitue un aspect central dans ma perspective ${ }^{5}$. Diessel (2005: 462) cite l'exemple suivant: «(3I) The sentence can only be assigned the right thruth condition, or alternatively be given the correct semantic representation, if the grammatical signifiance of "and" is taken into account». L'auteur donne ce commentaire: «In example (3I), for instance, it is the scalar particle only that indicates the occurrence of the following if-clause [...]». Ce qui m'intéresse dans cet exemple, c'est d'une part qu'if- $P$ est placé dans le champ de only; un tel cas de figure relève bien de cette partie i.2. D'autre part, l'opérateur only projette l'apparition de son opérande; autrement dit, only ouvre l'attente d'un segment qui sera placé sous sa portée ${ }^{6}$.

\subsection{Placer le constituant si $P$ en position frontale et dans le champ de l'adverbe seulement}

Soit les exemples [7] et [8]:

[7] - Les sportifs doivent-ils y manifester leur attachement aux droits de l'homme?

- Si et seulement si le mouvement olympique l'accepte, je suis d'accord pour que tous les sportifs portent le même badge.

(Corminboeuf - Corpus thèse : presse écrite, à propos des Jeux Olympiques)

[8] Ton poème ne pèche pas par la sécheresse, n'aie pas peur. C'est l'abondance au contraire qui peut causer de la fatigue. Tous ces détails «formant des ailes, servant de vestibule», etc., sont fastidieux. [...] Adopte donc nos coupures. Seulement si nous avons laissé des répétitions, corrige-les. Il y en avait dans le premier morceau (les hexamètres du commencement) que nous n'avons pas eu le temps de changer [...]. (Frantext: Gustave Flaubert, Correspondance, I852-1854, Paris, L. Conard, 1927, p. I13)

Ces exemples - a priori rarissimes en français - vont clairement à l'encontre des conclusions de Haiman (1978) et de toute hypothèse fonctionnaliste qui postulerait un rapport obligé entre le focus et le dernier terme d'un énoncé: le constituant si $P$ de [7] et [8] est en position frontale et il exprime pourtant un focus ${ }^{7}$. À propos

5. Dans l'étude de Söhrman, les exemples sont des deux types.

6. Un exemple du même type en français: «En tant que société globale, nous pouvons uniquement avoir du succès si nous tirons tous sur la même corde et si nous fonctionnons en équipe» (http://www.kmu. admin.ch/aktuell/00524/00525/00529/index.html?lang=fr).

7. Voir Lacheret-Dujour (2003) pour la situation symétrique des cas [7] et [8] : l'auteur analyse des exemples de circonstants positionnés en fin d'énoncé qui endossent une valeur de topique (sous une intonation "parenthétique»). Il serait intéressant d'avoir à disposition des faits oraux semblables aux exemples [7] et [8] afin de voir la façon dont est intonée la seconde partie de la structure. On pourrait peut-être attester deux schémas intonatifs distincts (intuitivement, il semble que l'on puisse lire les exemples [7] et [8] de deux façons différentes). 
d'exemples de ce type en espagnol, Montolío Durán (1999: 3705) soutient que c'est cette fonction de focus qui expliquerait l'impossibilité d'introduire la particule alors à l'initiale de l'élément $\mathrm{Q}$. L'auteur donne cet exemple: «Sólo si bace sol, iré

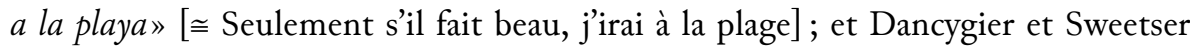
(2005: 225) donnent celui-ci, en anglais: "Only if you pay me \$20 will I mow the lawn» [气 Seulement si tu me paies $20 \$$ je tondrai la pelouse]. Verstraete (2004: 834) mentionne cet énoncé attesté, avec un when sous la portée de l'adverbe scalaire especially: "Especially when I speak in public I must show that I love all my sheep, like a good shepherd» [气 Surtout quand je parle en public je dois montrer que j'aime tous mes moutons, comme un bon berger]. Ces constructions sont peut-être plus communes en anglais et en espagnol qu'en français.

La singularité des tours en [7] et [8] est probablement liée au fonctionnement tout à fait particulier de seulement en français. D'autres circonstants - introduits par parce que dans l'exemple [9] et pour dans l'exemple [ı] - présentent en effet les mêmes caractéristiques:

[9] Georges: [...] Ne m'abandonnez pas. L'électricité se rallume.

Marie: Mon Dieu! Je reprends vie. Seulement parce que l'électricité se rallume, je reprends vie.

(Frantext: Henry de Montherlant, Demain il fera jour [1949], Paris, Gallimard, 196I, p. 744)

[io] HéLÈne et Faust : Arrête, arrête, pour l'amour de tes parents, tes désirs sans bornes! Sois tranquille, suis l'usage de tous!

EUPHORION: Seulement pour vous plaire, je m'arrêterai.

(Frantext: Goethe, Faust et le second Faust [1840], M. Allemand [éd.] et G. de Nerval [trad.], Paris, Garnier frères, 1956, p. 259)

Dans les dialogues en [9] et [ı。], le statut topical des éléments «je reprends vie» et «je m'arrêterai» est manifeste.

Je postulerai que les exemples [7] à [Io] ne sont pas des cas d'antéposition du circonstant, mais des cas de postposition du terme régissant (Q). De mon point de vue, c'est très différent: dans [7] à [ı], les modalités affectent le terme frontal, contrairement à ce qu'on observe dans les cas d'antéposition d'un complément. Ce phénomène fait penser à l'opération de «postfixation» observable en particulier à l'oral (Blanche-Benveniste et al., I990: 166) ${ }^{8}$. Ces circonstants ont donc un statut intraprédicatif, comme dans les exemples [3] à [6].

Le point commun des exemples [I] à [8] présentés supra est que l'élément si $P$ est intégré dans un dispositif ou placé dans le champ d'un opérateur - deux procédés focalisants. La portée de la négation ou de la restriction s'accompagne

8. En empruntant la terminologie de Blanche-Benveniste et al. (1990), je dirai que dans ce cas si $P$ est intégré au «noyau». 
très souvent d'une intégration de si $P$ à droite de $\mathrm{Q}$, comme dans les exemples [3] à $[5]$ - une position marquée pour les circonstants en si.

Il existe de nombreux cas de si $P$ placés à droite du terme $\mathrm{Q}$, mais qui sont détachés $(=\text { postposés })^{9}$. Dans ce scénario très différent des exemples [3] à [5], si $P$ exprime un topique:

[II] c'est une fille pas vraiment pétillante d'intelligence si tu vois ce que je veux dire (Corminboeuf - Corpus thèse: oral, cité par Jacqueline Authier-Revuz)

[I2] Je suis au bureau $305, s i$ t'as pas de clé.

(Corminboeuf - Corpus thèse: billet affiché sur une porte)

Les éléments si $P$ en [II] et [I2] ont un statut syntaxique différent des si $P$ des exemples [3] à [5] (voir Corminboeuf [2010] pour une étude des si $P$ de type «austinien»). Ils n'ont notamment pas le même comportement dans les cas d'enchâssement.

\section{Contraintes sur la position de si $P$ et contextes d'enchâssement}

Le critère que j’ai isolé dans la partie I est la capacité des propositions si $P$ focalisées à répondre positivement à la portée d'un opérateur (ou à l'insertion dans un dispositif). Cette propriété fait que le constituant si $P$ se comporte comme un élément intégré au prédicat. Cependant, tous les éléments si $P$ ne sont pas susceptibles de réagir positivement au clivage ou à la portée d'un opérateur. Les énonciations métaénonciatives comme «si tu as soif» dans «si tu as soif il y a de la bière dans le frigo» sont difficilement focalisables: «? c'est si tu as soif qu'il y a de la bière au frigo»/ «? il $y$ a de la bière au frigo seulement si tu as soif». Les exemples [II] et [I2] relèvent de cette catégorie. En fait, seuls les circonstants intraprédicatifs entrent dans des positions de focus, alors que les éléments si $P$ incidents à l'énonciation sont exclus dans ces positions (Corminboeuf, 2008, 2009, 2010).

Dans cette partie 2, j'utiliserai la propriété d'enchâssement comme un test révélant un certain nombre de contraintes liées à la stratification informationnelle. Tout d'abord, il y a une observation tendancielle de Ford et Thompson (1986: 359) sur l'anglais, que mon corpus vient confirmer: "When a conditional clause occurs within a nominalization, an infinitive, or a relative clause, there is a tendency for it to occur in final position». En effet, les structurations $\{Q$ si $P\}$ sont très bien

9. Ford et Thompson (1986: 367-368) présentent des exemples où l'élément if- $P$ postposé reprend une information du contexte proche, ce qui défocalise clairement if- $P$. Rousseau (I993: 258) parle de «thème postposé» pour les exemples de type $\{Q$ si $P\}$. Schiffrin (1992: 193) sur l'anglais et Söhrman (2002) sur l'espagnol observent que les postposées en si peuvent endosser une fonction de topique ou de focus. Montolío Durán (I996: 337 sq.) analyse les rendements en espagnol de la postposition de l'élément si $P$, en distinguant les cas où si $P$ est incorporé ou non dans le même contour intonatif que $Q$. 
représentées dans les enchâssements, ce qui tend à montrer que la position de si $P$ est sensible à un tel contexte.

Avant d'étudier ces contextes d'enchâssement (infra, 2.I et 2.2), je présenterai deux contraintes qui ne sont peut-être pas directement liées à des impératifs de stratification informationnelle: (i) la longueur des propositions et (ii) la portée du cadre ouvert par si $P$. Mais comme ces aspects entrent également en ligne de compte dans le positionnement de si $P$, il me paraît important de leur faire un sort.

(i) La longueur respective des propositions peut expliquer la position de si $P$ à l'intérieur d'une proposition que $\mathrm{P}$ :

[13] Très grossièrement (nous y reviendrons plus bas), on peut dire qu'un récit est un récit de fiction $s i$ ce discours représente des individus agissant sur des objets dans des situations, alors que le locuteur croit que ces individus, ces objets et ces situations n'existent pas ou n'existent pas tels qu'il les décrit.

(Corminboeuf - Corpus thèse: discours scientifique)

[14] Et alors, je pensais que, si j'osais prendre l'avion pour l'Égypte, je pourrais visiter les grands monuments que j'adore depuis longtemps.

(Corminboeuf - Corpus thèse: Alberto Giacometti, Écrits)

L'exemple [13] contient un élément $\mathrm{P}$ «à rallonge» qui comporte nettement plus de syllabes que Q. En plus, il s'agit d'une définition: logiquement, le terme défini précède sa définition. Dans l'exemple [I4], c'est la proposition Q qui est nettement plus longue que si $P$; le constituant si $P$ est antéposé à l'intérieur de l'enchâssement.

Ford et Thompson (1986:36r et 367) soulignent, en anglais, cette «tendency of writers to avoid initial dependent clauses which are disproportionately long with respect to their associated main clauses». L'une des stratégies de linéarisation établie par Berrendonner (1987) - dans une étude consacrée aux compléments régimes - est de placer les compléments courts avant les longs. Dans mes exemples, la proposition courte est ordinairement positionnée avant la proposition longue, au sein de l'enchâssement.

(ii) Par le biais du détachement, deux séquençages distincts de la structure argumentale de l'exemple [is] peuvent être observés:

[I5] Les cafetiers alémaniques craignent $<$ de se retrouver dans les chiffres rouges s'ils n'augmentent pas le prix de la tasse de café >.

(Corminboeuf - Corpus thèse: web)

Ce qui est en jeu ici, c'est l'expansion de l'opérande $y$ dans craindre ( $y x)$. Dans [I5], l'opérande est marqué par les parenthèses angulaires: la condition n'affecte que «de se retrouver dans les chiffres rouges». En antéposant si $P$, l'opérande se voit restreint au syntagme prépositionnel et le procès craindre ( $y x$ ) est entièrement inclus dans un espace fictif: 
[I'] S'ils n'augmentent pas le prix de la tasse de café, les cafetiers alémaniques craignent $<$ de se retrouver dans les chiffres rouges $>$.

(ex. modifié)

Le détachement a par conséquent une incidence sur la portée du cadre si $P$, puisque dans [I5'] c'est la crainte qui est mise sous condition. Les exemples d'enchâssement montrent que la portée locale ou globale de si $P$ joue un rôle dans l'ordonnancement des constituants.

Venons-en maintenant à la contrainte d'enchâssement. On peut faire l'hypothèse que pour être focalisé, le constituant si $P$ doit répondre négativement à une extraposition hors de l'enchâssement. Quand l'élément si $P$ peut sortir de l'enchâssement, il n'est pas focalisable.

\subsection{Format $[\mathrm{V} \text { que }\{Q \text { si } P\}]_{\mathrm{SV}}$}

Premier cas de figure: l'élément si $P$ est non antéposable, même au sein de l'enchâssement. Dans l'exemple [ı6], une antéposition de si $P$ est exclue:

[16] Quand on se porte bien, on admire comment on pourrait faire si on était malade; quand on l'est, on prend médecine gaiement: le mal y résout. On n'a plus les passions et les désirs de divertissements et de promenades, que la santé donnait [...].

(Corminboeuf - Corpus thèse: Blaise Pascal)

[16] ? On admire comment, si on était malade on pourrait faire. $\rightarrow$ antéposition à l'intérieur de l'enchâssement (ex. modifié)

[16"] ? Si on était malade, on admire comment on pourrait faire. $\rightarrow$ antéposition en dehors de l'enchâssement (ex. modifié)

Certes, une circonstancielle cadrative extraposée en quand occupe déjà la position frontale, ce qui rend peu praticable une seconde antéposition. Mais même sans la proposition quand $P$, il est pour ainsi dire impossible d'antéposer le constituant si $P$. En effet, le déplacement du circonstant, qui se comporte ici comme un complément valenciel, entraînerait un emploi absolu du verbe régissant («pourrait faire»). Même à l'intérieur de l'enchâssement, l'antéposition parait bloquée (voir [I6']). L'élément si $P$ est en quelque sorte intégré dans le prédicat du terme $\mathrm{Q}$ (voir partie I, supra).

Second cas de figure: l'élément si $P$ est antéposable au sein de l'enchâssement (mais pas en dehors). Les faits présentés sous ce point sont en contexte de discours rapporté ${ }^{10}$. La structure de rection particulière des verbes de parole fait qu'il est parfois difficile de démêler les cas de rection faible (Blanche-Benveniste et Willems, 2007) des «vrais enchâssements». Considérons l'exemple [I7]:

10. Il s'agit d'une observation statistique sur mon corpus et non d'un critère. 
[17] Le président iranien a à nouveau contesté la légitimité de l'État d'Israël. Il a proposé que l'Allemagne et l'Autriche l'accueillent sur leur territoire s'ils s'estiment coupables de massacres de Juifs pendant la Deuxième Guerre mondiale.

(Corminboeuf - Corpus thèse : web)

[I7'] Le président iranien a à nouveau contesté la légitimité de l'État d'Israël. Il a proposé que si l'Allemagne et l'Autriche s'estiment coupables de massacres de Juifs pendant la Deuxième Guerre mondiale, ils l'accueillent sur leur territoire.

$\rightarrow$ antéposition à l'intérieur de l'enchâssement

(ex. modifié)

[ı"'] ? Si l'Allemagne et l'Autriche s'estiment coupables de massacres de Juifs pendant la Deuxième Guerre mondiale, le président iranien a proposé qu'ils accueillent l'État d'Israell sur leur territoire.

$\rightarrow$ antéposition en dehors de l'enchâssement

(ex. modifié)

L'antéposition de si $P$ en dehors de l'enchâssement (exemple [ı7"]) est moins aisée qu'en [I $\left.{ }^{\prime}\right]$ supra. C'est peut-être lié au fait que le sujet est coréférentiel dans [Iร], ce qui n'est pas le cas pour les trois propositions de [I7] ${ }^{11}$. Mais ce qui joue surtout, c'est une propriété d'enchâssement dont je détaillerai le rôle après avoir présenté les exemples [18] et [19].

L'antéposition de si $P$ hors de l'enchâssement est aussi exclue dans [I8] :

[18] Son fils, ce gueux de Bébert, avait aussi disparu depuis le matin, et elle criait que ce serait un fameux débarras, s'il ne revenait plus.

(Frantext: Émile Zola, Germinal [1885], Paris, Gallimard, 1964, p. 1359)

[18'] Son fils, ce gueux de Bébert, avait aussi disparu depuis le matin, et elle criait que s’il ne revenait plus, ce serait un fameux débarras.

$\rightarrow$ antéposition à l'intérieur de l'enchâssement

(ex. modifié)

[r"] ? Son fils, ce gueux de Bébert, avait aussi disparu depuis le matin, et s'il ne revenait plus, elle criait que ce serait un fameux débarras.

$\rightarrow$ antéposition en dehors de l'enchâssement

(ex. modifié)

Pour [I8], une antéposition de l'élément si $P$ serait mieux tolérée après que $(=\mathrm{a}$ l'intérieur de l'enchâssement, voir [18']) qu'après $e t$ (= en dehors de l'enchâssement, voir [I8"]). Le constituant si $P$ fait partie du discours rapporté introduit par le verbe «criait».

11. L'intelligibilité de [ $\left.\mathrm{I}^{\prime}\right]$ est également peu optimale, probablement en raison de l'ordonnancement des anaphoriques. 
Dans l'exemple [19], le terme si $P$ est à l'intérieur d'une proposition que $P$ régie par une participiale:

[19] M. Sharon a qualifié de «présentation incorrecte de la situation» les propos indiquant que le bain de sang aurait été évité s'il ne s'était pas rendu jeudi au mont du Temple. (Corminboeuf - Corpus thèse : presse écrite)

[rg'] M. Sharon a qualifié de "présentation incorrecte de la situation» les propos indiquant que s'il ne s'était pas rendu jeudi au mont du Temple le bain de sang aurait été évité. $\rightarrow$ antéposition à l'intérieur de l'enchâssement (ex. modifié)

[ı"” ? S'il ne s'était pas rendu jeudi au mont du Temple M. Sharon a qualifié de «présentation incorrecte de la situation» les propos indiquant que le bain de sang aurait été évité.

$\rightarrow$ antéposition en dehors de l'enchâssement

(ex. modifié)

Comme pour l'exemple [I8], l'antéposition ne semble possible qu'au sein de l'enchâssement (voir [19']) ${ }^{12}$. Dans les exemples [I6] à [I9], si $P$ est par défaut en position de focus de la proposition enchâssée. La contrainte observée dans ces exemples est la suivante:

Une antéposition du membre si $P$ hors de l'enchâssement est peu praticable, parce que si $P$ est compris dans l'enchâssement, c'est-à-dire qu'il est incident à un élément qui est à l'intérieur de la proposition que $P$.

Autrement dit, quand si $P$ a une portée locale, quand il porte sur un élément qui est à l'intérieur de l'enchâssement (en l'occurrence Q), une extraposition est rendue difficile.

Mes observations convergent avec celles d'Hamon (2002) à propos de certaines constructions causales du français, et avec celles de Ford et Thompson $(1986: 366)$ à propos des «if-clauses» de l'anglais: «The qualification that this type of if-clause makes has a scope which is limited to an embedded clause».

Voyons pour comparaison l'exemple [2o] où le constituant si $P$ a une portée globale, c'est-à-dire qu'il n'est pas compris dans l'enchâssement:

[20] Que vous importera ensuite de savoir que je vous aime, si vous ne devez pas en être touchée et si vous ne m'accordez en retour que de la pitié?

$\rightarrow$ postposition de si $\mathrm{P}$

(Frantext: Édouard Estaunié, L'ascension de M. Baslèvre [1919], Paris, Perrin, 1923, p. 146)

12. Auer (2000: 22) signale, en allemand oral, un cas de blocage de l'antéposition d'une construction en wenn enchâssée dans une relative. 
[20'] $S i$ vous ne devez pas en être touchée et si vous ne m'accordez en retour que de la pitié, que vous importera ensuite de savoir que je vous aime?

$\rightarrow$ antéposition de si $\mathrm{P}$

(ex. modifié)

[20"] ? Que vous importera ensuite de savoir que si vous ne devez pas en être touchée et si vous ne m'accordez en retour que de la pitié je vous aime?

$\rightarrow$ enchâssement de si $\mathrm{P}$

(ex. modifié)

Dans la série [20] à [20"]:

- l'élément si $P$ est forcément extraposé, comme le montrent les structurations en [20] et [20']: la séquence $\{Q$ si $P\}$ ne peut pas être liée;

- l'élément si $P$ ne peut pas être enchâssé, comme le montre l'exemple [20"].

Ces propriétés s'expliquent par le fait que si $P$ porte sur l'ensemble [V que $Q]$ et non sur le terme $\mathrm{Q}$ uniquement.

\subsection{Format $[\mathrm{V} \text { que }\{\text { si } P, Q\}]_{\mathrm{sV}}$}

47 Dans ce cas de figure, si $P$ exprime un topique:

[21] Se souvenir que si l'on disait Saint-Germain-des-Prés, c'est parce qu'il y avait des prés. (Frantext: Georges Perec, Espèces d'espaces, Paris, Galilée, 1974, p. 84)

[22] alors il faut expliquer au téléspectateur que $s i$ il voit des images / c'est pas que vous avez caché une caméra qui vous filme / c'est que vous allez refaire / un an plus tard / le même parcours avec euh votre votre ami \

(Corminboeuf - Corpus thèse : oral, télévision, à propos d'alpinisme)

Dans [2I] et [22], il s'agit d'un type d'hypothétiques dites «factuelles» (Stage, 1991; Corminboeuf, 2013), qui présente un ordonnancement contraint : si $P$ précède systématiquement $Q$. La variante «c'est parce qu'il y avait des prés si l'on disait Saint-Germain-des-Prés» (exemple [2I]) ne semble en effet pas attestée en français. Certains circonstants en si ne peuvent donc qu'exceptionnellement être focalisés. Dans des constructions comme [2I] et [22], l'élément si $P$ ne peut jamais être intégré à droite, et ne peut que très rarement être postposé ${ }^{13}$. Pour [22], il faut en outre composer avec la longueur de $\mathrm{Q}$, qui comporte nettement plus de syllabes que $\mathrm{P}$, et avec le fait qu'il y a deux focus antagonistes (c'est pas que x... c'est que y), ce qui justifie qu'ils soient placés en fin d'énoncé.

13. Voici un exemple d'hypothétique «factuelle» tiré d'un article de linguistique, où l'élément si $P$ est exceptionnellement postposé: «Contrairement à ce que pense un certain nombre de linguistes américains, il ne faut pas identifier le support de l'énoncé à un élément d'information qui n'est pas nouveau pour l'interlocuteur, s'il est fréquent que le support reprenne des informations de la phrase précédente» (discours scientifique). Il s'agit d'un genre de «factuelle» (ici à valeur concessive) différent de [2I] et [22]. 


\subsection{Synthèse}

Les observations en rapport avec cette propriété d'enchâssement peuvent être résumées en trois points.

(i) Pour être focalisé, l'élément si $P$ ne doit pas être incident à l'ensemble [ $V$ que $Q$ ], mais doit avoir une portée restreinte à $\mathrm{Q}$; c'est une autre façon de dire que si $P$ doit être enchâssable. Par ailleurs, l'élément si $P$ doit être intégré à droite de $\mathrm{Q}$. Un corollaire est que si $P$ doit être de même rang syntaxique que $Q$. Le test de l'extraposition hors de l'enchâssement révèle cette exigence: dans [20], les segments "Que vous importera ensuite de savoir que je vous aime» et «si vous ne devez pas en être touchée et si vous ne m'accordez en retour que de la pitié» constituent des énonciations syntaxiquement autonomes. L'élément enchâssé je vous aime n'est pas de même rang syntaxique que l'énonciation si $P$, contrairement aux exemples [I6] à [ig]. Lorsque le membre si $P$ porte sur l'ensemble [ $V$ que $Q]$, comme dans [20], il est forcément détaché (quel que soit son emplacement) et son antéposition hors de l'enchâssement est possible.

(ii) À l'intérieur de l'enchâssement, les circonstants si $P$ détachés à gauche ont une fonction topicale (exemples [14], [2I], [22]). Antéposer si $P$ au sein de l'enchâssement semble être un moyen de lever une éventuelle ambiguïté. Dans cette position, si $P$ ne peut en effet pas avoir une incidence globale sur l'ensemble [Vque $Q$ ], i. e. sur un élément qui est en dehors de l'enchâssement.

(iii) Le troisième enseignement est qu'il existe des circonstants en si qui ne peuvent pas être topicalisés, comme dans [16] et [24] infra ${ }^{14}$, et qu'il en existe d'autres au contraire, comme en [2I] et [22], qui ne peuvent pas être focalisés. Les propositions si $P$ incidentes à l'énonciation (non enchâssables, toujours détachées), comme en [II], [I2] et [20], relèvent également du second cas de figure ${ }^{15}$.

Si les contextes d'enchâssement semblent favoriser la focalisation de si $P$, je ne considérerai pas qu'il s'agit là d'une opération de focalisation contrainte par la syntaxe. Ces faits révèlent plutôt les conditions que doit remplir un élément si $P$ pour pouvoir être focalisé.

\section{Le traitement des ellipses}

Cette partie 3 est centrée sur des constructions en si où le terme Q est ellipsé. Je fais l'hypothèse qu'il s'agit d'un procédé au service de la focalisation de si $P$. Avant d'étudier ces propositions si $P$ «tronquées», voyons les exemples [23] à [25].

La construction [23] montre que la portée d'un opérateur (partie I) et une ellipse du terme Q (partie 3) peuvent être cumulées:

14. Ceci a des incidences méthodologiques lorsqu'on fait des comptages à propos de la position de si $P$.

15. Les éléments si $P$ des exemples [II], [I2] et [20] ne sont pas des circonstants: ils forment des énonciations syntaxiquement autonomes (Corminboeuf, 2008, 2009, 2010). 
[23] l'engagement personnel est total / car l'avion ne viendra me rechercher que dans trois semaines / et seulement si les conditions météo le permettront \

(Corminboeuf - Corpus thèse : oral, télévision, à propos d'alpinisme)

Dans l'exemple [23], l'élément si $P$ est dans le champ de l'adverbe restrictif seulement et présente après et une ellipse du terme $\mathrm{Q}$ (il viendra me chercher) - tout comme dans les exemples analysés infra. L'ellipse de $Q$ peut être conçue comme l'auxiliaire d'une promotion informationnelle de si $P$, hypothèse que je reconduirai pour les exemples [26] et suivants.

Les comparatives comme [24] contiennent des ellipses très grammaticalisées:

[24] Au sens strict, un nom propre ne peut avoir de pluriel; ce serait aussi inconcevable que $s i$ le pronom «je» avait un pluriel.

(Corminboeuf - Corpus thèse: discours scientifique)

[24'] Au sens strict, un nom propre ne peut avoir de pluriel; ce serait aussi inconcevable que $<$ ce serait inconcevable $>$ si le pronom «je» avait un pluriel.

(ex. modifié)

Le constituant ellipsé «ce serait inconcevable» (= Q) étant prévisible (voir [24']), on peut en faire l'économie sans risque de carence d'informativité. L'élément si $P$ de l'exemple [24] ne peut pas être antéposé: il s'agit d'un circonstant à place fixe.

On pourrait décrire de la même façon les comparatives en comme si telles que celle de l'exemple [25]:

[25] Quand je ne souffre pas, me trouvant entre deux périodes de souffrance, je vis comme si je ne vivais pas.

(Henri Michaux, La vie dans les plis, Paris, Gallimard, 1972, p. 98)

[25'] Quand je ne souffre pas, me trouvant entre deux périodes de souffrance, je vis comme $<$ je vivrais $>$ si je ne vivais pas.

(ex. modifié)

Renchon (1967: 69) cite cet exemple de Maurois où l'élément Q («je vous verrais») est réalisé segmentalement: «J'essaie de vous voir comme je vous verrais si je ne vous aimais pas» ${ }^{16}$.

16. Certaines consécutives en tellement... que... - non elliptiques celles-ci-manifestent également un ordre contraint. Dans l'exemple suivant, le circonstant «si je me mettais à leur place» ne peut évidemment pas être placé avant l'adverbe «tellement»: «mais je sais que y a tellement d'astronautes derrière moi / y compris beaucoup d'Européens qui ont beaucoup de talent / euh qui sont prêts à à à effectuer des missions de longue durée dans la station / que je ne me sentirais pas à l'aise en fait / euh si je me mettais à leur place \» (oral, télévision, à propos de conquête spatiale). 
Les constructions [24] et [25] présentent un type particulier d'enchâssement, et à ce titre, sont concernées par la contrainte observée en partie 2. Ces tours enchâssent des complexes $\left\{\varnothing_{Q}\right.$ si $\left.P\right\}$.

\subsection{Les contextes de «coénonciation»}

L’exemple [26] est emprunté à Jeanneret (1999):

[26] LI: il faut absolument respecter le mycélium

L2: avec ça on aura des champignons l'année prochaine

$\mathrm{L}_{3}: s i$ la saison le permet

(Corminboeuf - Corpus thèse : oral)

$\mathrm{L}_{3}$ peut faire l'économie de l'élément $\mathrm{Q}$ parce que celui-ci est aisément restituable à partir du contexte antérieur.

\subsection{Entre effets d'inachèvement et conventionnalisation de l'ellipse}

Plusieurs classes de structures présentent des propositions si $P$ «tronquées», dans un contexte monologal ( $v$ interactionnel dans l'exemple [26], supra). Dans un premier temps, je liste quelques exemples (3.2.I). Dans un second temps, je rends compte de la façon dont ces constructions sont décrites dans le champ scientifique (3.2.2). Enfin, je précise la position que j’adopte (3.2.3).

\subsubsection{Les observables}

Voici un aperçu des constructions en question ${ }^{17}$ :

[27] Romantique et spontané, grand sportif également, il attache beaucoup d'importance à la beauté intérieure surtout et au charme d'une femme. Si vous êtes jeune, sensible..

(Corminboeuf - Corpus thèse: annonce matrimoniale)

Parfois, un inachèvement est suggéré au moyen de la ponctuation, comme cela semble être le cas des points de suspension dans l'exemple [27]; un point d'exclamation peut remplir le même office ${ }^{18}$. Mais de nombreux exemples ne présentent pas de telles marques:

17. On peut se reporter à Renchon (1967: 70-75) et Henry (1977) pour un inventaire de ces constructions. Je ne signale ici que des exemples écrits. Pour des faits oraux de ce genre en français, voir Debaisieux et al. (2008); en anglais oral: Ford (1997); en italien oral: Lombardi Vallauri (2004). Les résultats des contributions récentes de Patard (à paraître) et de Saez (à paraître) qui analysent finement certaines des classes de structures mentionnées ici, n'ont pas pu être intégrés à cet article.

18. Voir Renchon (1967: 90-98) et Moline (2010) pour une analyse de tours exclamatifs du type «Si tu savais comme elle a souffert!»; l'exemple [29] est un cas de figure voisin. À l'oral, c'est parfois l'allongement du progrédient qui est l'indice d'inachèvement. 
[28] C'est que s'il n'y a personne après vous, s'il n'y a personne qui sorte de vous; si personne n'est là pour vous continuer. S'ils s'en vont tous ainsi, il se dit; d'une façon ou de l'autre, s'ils s'en vont et s'ils m'abandonnent.

On s'est condamné à une seule chose, toujours la même, dans le même lieu: alors $s i$ on s'était trompé, parce qu'on croyait à son travail, mais peut-être qu'il vous a menti.

Si c'était une punition, et les autres vont courir le monde.

C'est pourquoi il garde son outil levé, réfléchissant [...].

(Charles-Ferdinand Ramuz, Passage du poète, Lausanne, L'Âge d'Homme, 199o, p. 28-29)

[29] «Mon enfant, si vous saviez avec quelle frivolité les peuples sont gouvernés», a dit le chancelier Axel Oxenstierna.

(Corminboeuf - Corpus thèse: presse écrite)

[30] Si le soleil ne revenait pas

(Corminboeuf - Corpus thèse : titre d'un roman de Charles-Ferdinand Ramuz)

[3I] Si c'est votre anniversaire

(Corminboeuf - Corpus thèse: presse écrite, titre de rubrique)

L'effet suspensif tend à disparaittre, mais le terme $Q$ demeure assez aisément restituable par inférence. Ainsi, le titre du roman de Ramuz en [30] conduit à inférer une partie $Q$ du genre «que se passerait-il?». Le titre de presse en [3I] est cataphorique par rapport au contenu de la rubrique en question.

Enfin, certains constituants si $P$ tendent à s'autonomiser et à se ritualiser. Cela semble être par exemple le cas pour les suggestions en [32a] et [32b] ainsi que pour les optatives en [33a] et [33b]:

[32a] Et si on reparlait automobile

(Corminboeuf - Corpus thèse : publicité)

[32b] Si nous ouvrions une bouteille, qu'en dirais-tu?

(Corminboeuf - Corpus thèse : cité dans la Grammaire Larousse)

[33a] «Pourquoi est-ce que j’y suis venu? Elle ne voulait pas que je monte. Si seulement je l'avais écoutée» - pendant qu'il allait toujours sans savoir où il allait.

(Frantext: Charles-Ferdinand Ramuz, La grande peur dans la montagne [1926], Paris, Grasset, 1942, p. 97)

[33b] Si je pouvais dormir.

(Frantext: Samuel Beckett, En attendant Godot, Paris, Minuit, 1952, p. 118)

En $[32 b]$, ce n'est peut-être pas un hasard si c'est l'exemple tiré d'une grammaire qui comprend la structure «complète» \{protase + apodose\}. Mais pour la plupart 
des constructions citées ci-dessus, les versions $\{$ si $P, Q\}$ sont assez bien représentées. C'est le cas par exemple pour les propositions en si seulement:

[34] Je me lève en sursaut: si seulement je pouvais m'arrêter de penser, ça irait déjà mieux. (Frantext: Jean-Paul Sartre, La nausée, Paris, Gallimard, 1938, p. 130)

La protase de l'exemple [35] ci-dessous pourrait s'autonomiser pour fonctionner comme une exclamative: «Si vous saviez comme nous nous aimons!».

[35] Si vous saviez comme nous nous aimons, vous en seriez jaloux.

(Corminboeuf - Corpus thèse: $\mathrm{M}^{\mathrm{me}}$ de Sévigné)

\subsubsection{Le traitement des propositions si $\mathrm{P}$ «tronquées » dans les travaux de linguistique}

Ces propositions si $P$ isolées tendent à être traitées comme des «indépendantes»: c'est l'hypothèse d'Henry (1977), dans un article paru une première fois en 1956 et intitulé «Les propositions introduites par si en fonction d'indépendantes». Evans (2007), Stirling (1999) et Lombardi Vallauri (2004) font des observations convergentes ${ }^{19}$.

Pour Stirling (1999), qui travaille sur l'anglais d'Australie, il y a des constructions elliptiques et des structures réellement isolées. Les propositions indépendantes sont celles à valeur directive (i. e. un ordre atténué, comme «si tu veux bien te pousser de côté») ou à valeur optative (si seulement $P$ ). Les «indépendantes» présentent les propriétés suivantes: (i) elles ont un contour prosodique complet; (ii) il est difficile d'y voir une ellipse, celle-ci n'étant pas aisément reconstituable; (iii) elles fonctionnent comme des "propositions principales». Stirling (1999: 292) traite ce phénomène en termes de "grammaticalisation», en reprenant à Evans (2007: 370) les principes d'un développement diachronique en quatre étapes, dont ces «indépendantes» seraient le produit de sortie ${ }^{20}$ :

- complexe \{proposition subordonnée + proposition principale\};

- ellipse de la proposition principale, reconstituable en contexte;

- conventionnalisation de l'ellipse, c'est-à-dire restriction de l'interprétation du matériau ellipsé;

- conventionnalisation de l'emploi d'une «subordonnée» dans un rôle de "principale», ce qui entraîne une restauration malaisée du matériau ellipsé et une spécialisation sémantique.

Stirling emprunte à Evans la notion d'insubordination, qui correspond peu ou prou à la réanalyse (Patard, à paraître) d'une proposition subordonnée isolée en une construction indépendante.

19. On trouve ces structures dans de très nombreuses langues, selon Stirling, Lombardi Vallauri et Evans. Henry (1977) souligne que plusieurs types de propositions si $P$ «tronquées» étaient attestés en ancien français.

20. Il semble que le texte d'Evans circule depuis longtemps dans la communauté scientifique et que Stirling ait eu accès au manuscrit bien avant sa parution en 2007. 
À partir d'un corpus d'italien parlé, Lombardi Vallauri (2004) analyse les valeurs sémantiques des propositions $s e-P$ qu'il traite également comme des «indépendantes». L'auteur considère que ces constructions ont au moins deux patrons intonatifs distincts: soit «conclusif», soit «suspensif» ${ }^{21}$. Le contenu ellipsé peut être ramené à quelques types génériques, que je reproduis ici dans une version française correspondante.

- Emplois polémiques: si $P<$ ce que tu viens de dire est non pertinent $>$ :

[36] LI: Demain après-midi j'irai voir l'exposition.

L2: Mais si tu as un rendez-vous chez le dentiste.

(je traduis un exemple de Montolío Durán, 1999: 368I)

- Requêtes: si $P<$ ce serait bien $>$ :

[37] Si tu pouvais passer chez moi demain...

(ex. fabriqué, inspiré de ceux de Lombardi Vallauri, 2004)

- Questions: si $P<$ qu'arrivera-il ? > :

[38] Mais, Seigneur, cependant s'il épouse Andromaque?

(Frantext: Jean Racine, Andromaque [1697], Paris, Hachette, I886, p. 72)

- Emplois pour «rassurer» l'allocutaire: si $P<$ pourquoi se tourmenter > :

[39] Si je te dis de ne pas t'inquiéter...

(ex. fabriqué, inspiré de ceux de Lombardi Vallauri, 2004)

- Ménagement des faces: si $P<$ faites-le $>$ :

[40] Si mon colonel veut se donner la peine d'entrer...

(Corminboeuf - Corpus thèse: Alphonse Allais, Félix Galipaux et Paul Bonhomme, Monsieur la Pudeur)

- Suggestions: si $P<$ qu'en dirais-tu? $>$ :

[4I] Si on jouait au jeu du cadavre exquis, Histoire d'nous passer un peu notre ennui

(Corminboeuf - Corpus thèse: Serge Gainsbourg, «Le cadavre exquis»)

Les constructions en se de l'italien fonctionneraient de manière autosuffisante et l'absence du terme $\mathrm{Q}$ serait sans dommage pour l'intercompréhension. Selon Lombardi Vallauri (2004), la fonction sémantico-pragmatique de la principale se voit en quelque sorte incorporée dans l'«indépendante» si $P$.

21. Voir Henry (1977) et Debaisieux et al. (2008) pour des hypothèses et des observations sur la prosodie de ces structures en français. 
Les travaux de Stirling (1999), Lombardi Vallauri (2004) et Evans (2007) appellent deux commentaires.

I. Pour eux, une ellipse conventionnalisée n'est plus une ellipse. Or, à mon sens, c'est une caractéristique de l'ellipse que de se conventionnaliser, de présenter des restrictions sémantiques, etc. Selon ces auteurs, il y aurait ellipse seulement quand celle-ci est récupérable dans le discours antérieur.

2. Il est généralement postulé qu'une proposition subordonnée implique la coprésence d'une proposition principale. Or, dans ces exemples du type $\left\{\right.$ si $\left.P \varnothing_{Q}\right\}$, seule la subordonnée est verbalisée, ce qui conduit les auteurs à conclure que ce ne peut être qu'une proposition «indépendante».

Dans un cadre théorique très différent, Debaisieux et al. (2008) font l'hypothèse que ces constituants si $P$ se répartissent en deux classes, les pseudo-raccourcis et les raccourcis. Le concept de «raccourci» serait pour les auteurs un moyen de se passer de celui d'«ellipse». Les pseudo-raccourcis présentent un constituant si $P$ préfixé marqué par une intonation non conclusive, suivi d'un «noyau» non verbal (ou «paraverbal») ${ }^{22}$ :

[42] mais bon $s i$ tu me dis que tu as l'ordinateur //

(oral, cité par Debaisieux et al., 2008: 225)

Le noyau - siège du focus - serait à reconstituer par inférence, à partir du contexte. De leur côté, les raccourcis présentent un élément si $P$ qui fonctionne comme un «noyau»; il manifeste un effet de focalisation, une force illocutionnaire propre et une autonomie communicative :

[43] mais si tu savais ce que moi j'étais contente $\backslash$

(oral, cité par Debaisieux et al., 2008: 243)

Il s'agirait de constructions \{préfixe + noyau\} dans lesquelles «le noyau reconstruit par inférence a été conventionnellement intégré dans la représentation sémantique» du préfixe (Debaisieux et al., 2008: 236). La reconstruction du noyau - conventionnalisée - se fait sans recourir au contexte. Le contour prosodique observé dans les raccourcis est celui d'un énoncé assertif. Cette étude sur le français suggère deux commentaires.

I. Dans les pseudo-raccourcis, le noyau - élément en principe «indispensable»peut être non verbal. Selon les auteurs, le focus serait logé dans un élément absent segmentalement (le noyau).

22. Dans la macrosyntaxe utilisée par l'équipe du GARS-DELIC (Groupe aixois de recherche en syntaxe - Description linguistique sur corpus), le noyau est un élément indispensable à la construction d'un énoncé, qui manifeste une autonomie syntaxique et intonative; il est placé dans le champ des modalités d'énoncé. Le préfixe est un élément placé dans la périphérie gauche du noyau et ne bénéficiant pas d'une autonomie communicative. 
2. Le gain théorique obtenu en substituant la notion de raccourci à celle d'ellipse serait à questionner. Tout dépend de la théorie de l'ellipse dont on se dote.

\subsubsection{Une proposition de description}

Je retiendrai essentiellement deux points des études dont j'ai brièvement rendu compte.

I. Le fait qu'il y aurait un différentiel de grammaticalisation dans ces structures (Henry, 1977; Evans, 2007) : certaines sont ressenties comme elliptiques («suspensives»), d'autres comme des propositions «indépendantes» («conclusives»). Certaines observations syntactico-prosodiques et sémantiques fondent cette distinction.

2. L'idée que dans une partie des exemples, les caractéristiques fonctionnelles du «noyau» ou de la proposition «principale» sont reportées sur le constituant si $P$.

Pour le français, il me semble que la grande majorité des propositions si $P$ «tronquées» peuvent être ramenées aux structures bipartites, même lorsque ni l'intonation, ni la ponctuation n'induisent un effet suspensif ${ }^{23}$. Mais il y a clairement des constructions plus ritualisées que d'autres.

Je ferai l'hypothèse que, dans les exemples [26] à [34], l'ellipse de $Q$ est rendue possible parce que c'est si $P$ qui est le focus, ce qui concorde assez bien avec l'observation 2 que je viens de formuler ci-dessus ${ }^{24}$. Seuls les éléments récupérables, c'est-à-dire ceux dont on peut faire l'économie, sont ellipsables. En conséquence, les ellipses identifiées dans les exemples ci-dessus peuvent être envisagées comme des indices de la focalisation de si $P$.

Par comparaison avec les auteurs cités supra, je ferai une utilisation moins spécifique de la notion d'«ellipse». Je n'entends pas par «ellipse» la reconstruction fidèle d'une structure de départ éventuellement pré-assertée, mais la catalyse d'un contenu informationnellement prévisible. La notion de catalyse est due à Hjelmslev (1968: I29 sq.) (cité par Reichler-Béguelin, 1997: 37), qui donne l'exemple de la préposition sine qui régit un ablatif. $\mathrm{Si}$, pour une raison quelconque, le nom à l'ablatif est absent, celui-ci peut être catalysé à partir de sine, la préposition impliquant la cooccurrence d'un régime. Dans mes exemples - du moins dans ceux où l'élément si $P$ est clairement régi par un terme $\mathrm{Q}$ ellipsé -, on pourrait dire que c'est l'élément impliqué (si $P$ ) qui permet de catalyser un élément impliquant. Le membre régissant $\mathrm{Q}$ peut donc être conjecturé en contexte ou par convention à partir d'une classe de solutions possibles. Le terme régissant est récupérable par catalyse à partir du terme régi. Cette analyse m'évite de parler de propositions si $P$

23. De mon point de vue, seule la lexie s'il vous plaît et, à la rigueur, les constructions en si seulement pourraient être rangées au stade 4 d'Evans (2007).

24. Rothenberg (1989: I5I) considère que dans « $\mathrm{Si}$ on y allait?» la suggestion est le rhème, alors que le thème est constitué par la question et «l'idée d'y aller». 
«indépendantes», puisque le phénomène d'ellipse observé n'implique pas que Q soit absent au plan conceptuel, il est simplement non réalisé segmentalement (l'ellipse est un procédé d'économie).

En guise de conclusion à cette partie 3, voyons encore le dialogue en [44], où tout est ellipsé sauf le relateur $s i$ :

[44] Philippe, irrité, répliqua: «Si j’entre dans la Laconie, je vous en chasserai tous». Ils lui répondirent: $« S i »$.

(Jean-Jacques Barthélemy, cité par Émile Littré, Dictionnaire de la langue française, Paris, L. Hachette, I873-1874, art. «Si», p. 1929)

Dans «Si j'entre dans la Laconie, je vous en chasserai tous», l'élément si $P$ a une fonction de topique par défaut. La réponse - qui se comprend au sens de «encore faut-il que tu entres dans la Laconie»-déplace le focus sur le relateur si. Les constituants ellipsés, $\mathrm{P}$ et $\mathrm{Q}$, sont facilement récupérables.

\section{Conclusion}

Dans un premier temps (partie I), j'ai étudié les moyens variés à disposition des locuteurs pour focaliser les circonstants en $s i$, en particulier la portée d'un opérateur. J'ai montré que l'intégration ou le détachement de si $P$ et sa position (à gauche ou à droite) contribuent au marquage de la stratification informationnelle. Dans un second temps (partie 2), j'ai analysé des cas où les circonstants en si présentent des contraintes d'ordonnancement des propositions comme cette propriété de contiguïté dans les contextes d'enchâssement, qui stipule que si $P$ ne peut être antéposé que conjointement à l'élément auquel il s'applique, en l'occurrence le terme Q. Dans un troisième temps (partie 3), j'ai proposé une analyse des cas où il y a ellipse - plus ou moins conventionnalisée - du membre $\mathrm{Q}$, opération qui semble s'accompagner d'une focalisation du constituant si $P$.

\section{Références}

Akatsuka, N. 1986. Conditionals are Discourse-Bond. In E.C. Traugott et al. (éd.), On Conditionals. Cambridge - Londres - New York - Melbourne: Cambridge University Press: 333-35I.

Auer, P. 200o. Pre- and Post-positioning of Wenn-Clauses in Spoken and Written German. Interaction and Linguistic Structures I5: I-36. En ligne à l'adresse suivante: http://www. inlist.uni-bayreuth.de/issues/15/inlistı5.pdf.

BerRendonner, A. 1987. L'ordre des mots et ses fonctions. Travaux de linguistique I4-15: 9-19.

Blanche-Benveniste, C. et al. 1990. Le français parlé: études grammaticales. Paris: Éditions du CNRS.

Blanche-Benveniste, C. et Willems, D. 2007. Un nouveau regard sur les verbes «faibles». Bulletin de la Société de linguistique de Paris 102 (I) : 217-254. 
Charolles, M. 2003. De la topicalité des adverbiaux détachés en tête de phrase. Travaux de linguistique 47 : $\mathrm{II}-5 \mathrm{I}$.

Comrie, B. 1986. Conditionals: A Typology. In E.C. Traugott et al. (éd.), On Conditionals. Cambridge - Londres - New York - Melbourne : Cambridge University Press : 77-99.

Corminboeuf, G. 2008. Entre détachement et intégration: la topographie des constructions en si et le marquage de la structure informationnelle. In J. Durand, B. Habert et B. LaKs (éd.), Actes du congrès mondial de Linguistique française (CMLF 2008). Paris : Institut de linguistique française : 2439-245I. En ligne à l'adresse suivante: http://www. linguistiquefrancaise.org/articles/cmlf/pdf/2008/or/cmlfo8042.pdf.

Corminboeuf, G. 2009. L'expression de l'bypothèse en français: entre bypotaxe et parataxe. Bruxelles: De Boeck-Duculot.

Corminboeuf, G. 2oio. Une description des constructions introduites par un si dit «austinien». Travaux de linguistique 60: I27-I44.

Corminboeuf, G. 20I3. Factualité et conditionnalité. In C. Norén et al. (éd.), Modalité, évidentialité et autres friandises langagières. Berne - Berlin - Bruxelles : P. Lang: 4I-60.

Cornulier, B. (de) 1985. Effets de sens. Paris: Minuit.

Dancygier, B. et Sweetser, E. 2005. Mental Spaces in Grammar: Conditional Constructions. Cambridge: Cambridge University Press.

Debaisieux, J.-M., Deulofeu, J. et Martin, P. 2008. Pour une syntaxe sans ellipse. In J.-C. Pitavy et M. Bigot (éd.), Ellipse et effacement: du schème de phrase aux règles discursives - Actes du colloque international de linguistique, 27-28 octobre 2005, Saint-Étienne. Saint-Étienne: Publications de l'Université de Saint-Étienne: 224-246.

Delaveau, A. 1980. Questions sur l'analyse de si. Linx 2: 7-34.

Diessel, H. 2005. Competing Motivations for the Ordering of Main and Adverbial Clauses. Linguistics 43 (3): 449-470.

Evans, N. 2007. Insubordination and Its Uses. In I.A. NikolaEva (éd.), Finiteness: Theoretical and Empirical Foundations. Oxford: Oxford University Press : 366-43I.

Ford, C. et Thompson, S. 1986. Conditionals in Discourse: A Text-Based Study from English. In E.C. Traugott et al. (éd.), On Conditionals. Cambridge - Londres - New York Melbourne: Cambridge University Press: 353-372.

Haiman, J. 1978. Conditionals Are Topics. Languages 54 (3): 564-589.

Hamon, S. 2002. Les conjonctions causales et la propriété d'enchâssement. Linx 46:25-35.

HENRY, A. 1977. Études de syntaxe expressive: ancien français et français moderne. Bruxelles: Éditions de l'Université de Bruxelles. Chapitre: Les propositions introduites par si en fonction d'indépendantes: 209-244.

Hjelmslev, L. 1968. Prolégomènes à une théorie du langage. Paris: Minuit.

JEANNERET, T. 1999. La coénonciation en français - Approches discursive, conversationnelle et syntaxique. Berne - Berlin - New York: P. Lang.

Kronning, H. 1996. Modalité, cognition et polysémie: sémantique du verbe modal «devoir». Uppsala - Stockholm: Acta Universitatis Upsaliensis - Almqvist \& Wiksell International.

LACHERET-Dujour, A. 2003. La prosodie des circonstants en français parlé. Louvain - Paris : Peeters.

Lombardi Vallauri, E. 2004. Grammaticalization of Syntactic Incompleteness: Free Conditionals in Italian and Other Languages. SKY Journal of Linguistics 17: 189-215. 
LORIAN, A. 1964. L'expression de l'bypothèse en français moderne: antéposition et postposition. Paris: Minard.

Moline, E. 2oro. De l'interrogation à l'exclamation: comme $P$ argument de savoir, $\mathrm{XVII}^{\mathrm{e}}-\mathrm{XX}^{\mathrm{e}}$ siècles. Lingvisticae Investigationes 33 (2):309-328.

Montolío Durán, E. 1996. Gramática e interacción (ensayo metodológico para el análisis del español conversacional). In A. Briz Gómez et al. (éd.), Pragmática y grámatica del español hablado. Valence - Saragosse: Universidad de Valencia - Libros pórtico: 329-34I.

Montolío Durán, E. 1999. Las construcciones condicionales. In I. Bosque et V. Demonte (éd.), Gramática descriptiva de la lengua española. Madrid: Espasa. Vol. 3 : Entre la oración y el discurso. Morfología: 3643-3738.

NøLKe, H. 1994. Linguistique modulaire: de la forme au sens. Louvain - Paris: Peeters - Société pour l'information grammaticale.

PATARD, A. à paraître. Réflexions sur l'origine de l'insubordination. Le cas de trois insubordonnées hypothétiques du français. Langages: numéro thématique intitulé Réanalyse et changement linguistique. M.-J. BÉGuelin, G. CoRminboeuf et L.A. JohnSEN (éd.). Paris : A. Colin.

REICHLER-BÉguelin, M.-J. 1997. Anaphores pronominales en contexte d'hétérogénéité énonciative: effets d'(in)cohérence. In W. De Mulder, L. Tasmowski-De Ryck et C. Vetters (éd.), Relations anaphoriques et (in)cohérence. Amsterdam - Atlanta: Rodopi : $3 \mathrm{I}^{-} 54$.

Renchon, H. 1967. Études de syntaxe descriptive. Bruxelles: Palais des Académies. T. I : La conjonction «si» et l'emploi des formes verbales.

Rossi, M. 1999. L'intonation, le système du français: description et modélisation. Paris - Gap: Ophrys.

Rothenberg, M. 1989. Quelques moyens syntaxiques de rhématisation et de thématisation en français. Bulletin de la Société de linguistique de Paris 84 (I) : I43-I6I.

Rousseau, A. 1993. L'implication logique dans la description et l'organisation des phénomènes de subordination. Travaux linguistiques $d u$ Cerlico 6: 227-263.

SAEz, F. à paraître. Découplage de constructions en quand. G. Corminboeuf, L.A. Johnsen et M.-J. BÉGUELIN (éd.).

SChiffrin, D. 1992. Conditionals as Topics in Discourse. Linguistics 30 (I) : 165-197.

Söhrman, I. 2002. Nadie protestaba si comía poco - La inversión de los períodos condicionales. Romansk Forum 16: 195-205.

StAGE, L. I99I. Analyse syntaxique et sémantique de la conjonction si dans les propositions factuelles. Revue romane 26 (2): 163-205.

StiRling, L. 1999. Isolated If-Clauses in Australian English. In P. Collins et D. LeE (éd.), The Clause in English: In Honour of Rodney Huddleston. Amsterdam - Philadelphie: J. Benjamins : 273-294.

SweEtser, E. 1990. From Etymology to Pragmatics: Metaphorical and Cultural Aspects of Semantic Structure. Cambridge - New York - Melbourne : Cambridge University Press.

Verstraete, J.-C. 2004. Initial and Final Position for Adverbial Clauses in English: The Constructional Basis of the Discursive and Syntactic Differences. Linguistics 42 (4): $819-853$. 